Tecnociencia. Vol. 22. $\mathrm{N}^{\circ}$ 2: 87-101

julio-diciembre 2020

\title{
CALLIPHORIDAE DE INTERÉS FORENSE ASOCIADAS A TRES CEBOS DE CERDOS DOMÉSTICO (Sus scrofa L.) EN UN ÁREA RURAL, CORREGIMIENTO DE LA PINTADA, PROVINCIA DE COCLÉ, PANAMÁ
}

\author{
1Percis A. Garcés \& ${ }^{2}$ Milexi Molinar \\ ${ }^{1}$ Universidad de Panamá, Vicerrectoría de Investigación y Postgrado, Programa \\ Centroamericano de Maestría en Entomología. \\ ${ }^{2}$ Universidad de Panamá, Vicerrectoría de Investigación y Postgrado, Programa \\ Centroamericano de Maestría en Entomología. \\ e-mail: ${ }^{1}$ perchysg@gmail.com, ${ }^{2}$ mymolinar@ hotmail.com
}

\section{RESUMEN}

La familia Calliphoridae habita en todas las regiones biogeográficas y comprende más de 1000 especies y 150 géneros a nivel mundial. Usualmente son el primer taxón en colonizar cadáveres frescos, pues su desarrollada capacidad para detectar olores olfato les permite colonizar cadáveres horas o minutos después del deceso, pudiendo encontrarse en todos los estados de descomposición cadavérica. El presente estudio se realizó durante la temporada lluviosa, en el mes de noviembre de 2017, en el corregimiento de La Pintada, provincia de Coclé. Se utilizaron vísceras de cerdo (Sus scrofa L.) correspondientes a cerebro, hígado, y corazón. De acuerdo con el análisis de correspondencias (CPA), las especies Chrysomya megacephala y Cochliomyia macellaria fueron las más abundantes; ambas están correlacionadas positivamente, debido a que sus poblaciones fueron las más capturadas y por ende pueden ser utilizadas como las principales indicadoras forenses. Lo opuesto ocurrió con las especies Chrysomya rufifacies, Lucilia sericata y Lucilia eximia, que registraron poblaciones bajas, y de acuerdo con el análisis tienen correlación negativa.

\section{PALABRAS CLAVES}

Entomología forense, vísceras, Chrysomya megacephala y Cochliomyia macellaria. 


\title{
CALLIPHORIDAE OF FORENSIC INTEREST ASSOCIATED TO THREE BAITS FROM DOMESTIC PIGS (Sus scrofa L.) IN A RURAL AREA, VILLAGE AT LA PINTADA, PROVINCE OF COCLE, PANAMÁ.
}

\begin{abstract}
The Calliphoridae family inhabits all biogeographical regions and comprises more than 1000 species and 150 genera in the world. Caliphorids are usually the first taxon to colonize fresh corpses since their developed sense of smell, allows them to colonize corpses minutes or hours after death. They can be found in all states of cadaveric decomposition. This study was conducted during the rainy season in november 2017, in the village of La Pintada, province of Coclé. Pig (Sus scrofa L.) viscera corresponding to the brain, liver and heart isused. According to the correspondence analysis (CPA), Chrysomya megacephala and Cochliomyia macellaria are correlated positively, since their populations were the most captured, so they can be used as main forensic indicators. The opposite occurred with species Chrysomya rufifacies, Lucilia sericata and Lucilia eximia, registered lower populations, and according to the analysis, their correlation was negative.
\end{abstract}

\section{KEY WORDS}

Forensic entomology, viscera, Chrysomya megacephala and Cochliomyia macellaria.

\section{INTRODUCCIÓN}

La biología y la ecología de este grupo es muy variada, siendo generalmente necrófagos, predadores, así como parasitoides de lombrices de tierra y caracoles y huéspedes de termiteros (Rognes, 1997), aunque algunas especies pueden ser vectores mecánicos de bacterias, protozoos y helmintos (Mariluis y Schnack, 2002).Otras tienen importancia médica y veterinaria, debido a que producen miasis a aves y mamíferos entre ellos al hombre (Zump, 1965; Guimarães $e t$ al., 1983), lo que explica su amplia distribución y extraordinaria capacidad de adaptación y colonización de nuevos hábitats.

Los Calliphoridae usualmente son el primer taxón en colonizar cadáveres frescos, pues su desarrollada capacidad para la detección de olores les permite colonizar cadáveres horas o minutos después de la 
muerte (Anderson y Van Laerhoven, 1996; Amendt et al., 2011), encontrarse en todos los estados de descomposición cadavérica (Wolff et al., 2001; Ordóñez et al., 2008;). Por ello, el rango de distribución de estos es frecuentemente utilizado por entomólogos forenses durante investigaciones de muertes (Byrd y Castner, 2010).

El grado y modo en que esta relación ocurre, depende de las características geográfico-climáticas de cada grupo humano, de su modo de vida y el hábitat a su alrededor, afectando las poblaciones de insectos (Nuorteva, 1963).

El uso de órganos o vísceras de animales permite evaluar de una manera rápida y efectiva la sucesión de la entomofauna y los fenómenos cadavéricos que ocurren de forma progresiva, lo cual contribuye a conocer cómo es la descomposición de los diferentes órganos y, como los insectos destruyen los mismos (Rodríguez y Salazar, 2014). Por lo que, este un método práctico, rápido y sencillo para lograr determinar la entomofauna de cualquier lugar, donde aparecen cadáveres expuestos y donde los peritos no cuentan con suficiente tiempo para reproducir la escena de muerte.

Por lo que, el propósito de este trabajo es establecer la preferencia de las especies de moscas Calliphoridae por los tres cebos (hígado, cerebro y corazón) de cerdos domésticos en descomposición, en un área rural de La Pintada.

\section{MATERIALES Y MÉTODOS}

\section{AREA DE ESTUDIO}

El estudio se realizó durante la temporada lluviosa, específicamente el mes de noviembre de 2017, en el corregimiento de La Pintada, provincia de Coclé, a una altura de 1.5 metros con coordenadas: $8^{\circ} 35^{\prime} 30.6^{\prime}, \mathrm{N}$ y $80^{\circ} 27^{\prime} 0.1^{\prime}$ 'O, situada en un área rural.

Se utilizaron vísceras de cerdos domésticos (Sus scrofa L.) correspondientes a cerebro, hígado y corazón. Los mismos se extrajeron y se mantuvieron en frío por periodo de tres días, para luego 
ser colocados en bolsas plásticas cerrada herméticamente antes de su exposición en campo.

Los muestreos se realizaron durante cinco días continuos, del 2 al 6 de noviembre 2017. Se utilizando trampas construidas de botellas plásticas, las cuales fueron sus pendidas en ramas de árboles y colocadas de forma triangular en el área de muestreo, a una distancia $50 \mathrm{~m}$ entre trampas.

A cada trampa se hicieron dos agujeros laterales para permitir la entrada de las moscas. Las tres botellas plásticas fueron cortadas a la mitad para colocar en su extremo inferior los respectivos órganos. Posteriormente ambas mitades fueron pegadas con "tape" adherente para evitar que las moscas atrapadas pudieran salir. En el fondo de cada trampa se colocó un envase plástico donde se colocaron las vísceras y más al fondo de la trampa se colocaron $350 \mathrm{ml}$ de alcohol al $70 \%$ para preservar a las moscas.

Cada día se procedió a revisar y a extraer las moscas de las trampas. Una vez extraídas estas fueron cambiadas a otros viales con alcohol al $70 \%$ para su preservación y rotuladas con la información correspondiente (\# de trampa, lugar y fecha de colecta y cebo). Las muestras fueron trasladadas al laboratorio del Programa Centroamericano de Maestría en Entomología (PCMENT), de la Universidad de Panamá, donde se separaron, cuantificaron y se montaron en alfileres entomológicos. Con la ayuda de un estereoscopio marca Leica Zoom 2000, se procedió a la identificación de las moscas, para lo cual se emplearon las claves de Whitworth, (2006) y Amat et al., (2008). También se tomaron los parámetros ambientales de temperatura y humedad relativa. Para determinar los grados de preferencia por los cebos se realizó un análisis de correspondencia (CPA), con el programa estadístico Past 3.

Durante el estudio la temperatura promedio fue de $28.3^{\circ} \mathrm{C}$ y la humedad relativa promedio de $70.3 \%$. 


\section{RESULTADOS}

Se capturaron un total de 619 individuos de Calliphoridae, distribuidos en tres géneros y cinco especies pertenecientes a las subfamilias Luciliinae y Chrysomyinae. Las especies más abundante fueron Chrysomya megacephala (Macquart, 1843) con 451 especímenes, seguidos de Cochliomyia macellaria (Fabricius, 1775) con 116 especímenes, Chrysomya rufifacies (Fabricius, 1794) con 23 especímenes, Lucilia sericata (Meigen, 1826) con 18 especímenes y Lucilia eximia (Wiedemann, 1819) con 11 especímenes (Figura 1).

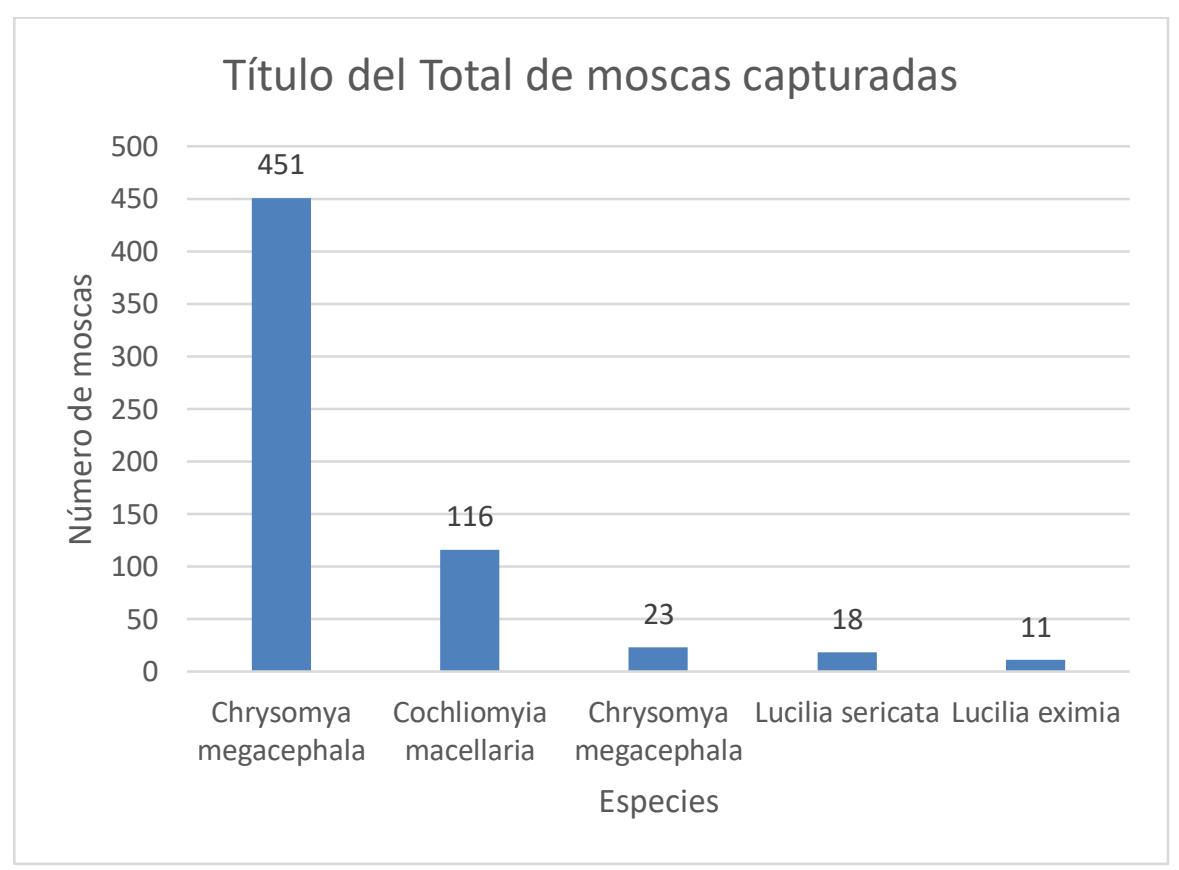

Fig. 1 Total de moscas capturadas

El cerebro que registró el mayor número de individuos con 290 especímenes, seguido del hígado con 208 especímenes y menor número el corazón, con 121 especímenes (Cuadro 1). 
Cuadro 1. Abundancia de especies en el área de estudio

\begin{tabular}{lcccccc}
\hline & \multicolumn{2}{c}{ Cerebro } & \multicolumn{2}{c}{ Hígado } & \multicolumn{2}{c}{ Corazón } \\
Especie & Ind. & $\%$ & Ind. & $\%$ & Ind. & $\%$ \\
\hline Chrysomya megacephala & 187 & 64.5 & 185 & 88.9 & 79 & 65.3 \\
Cochliomyi amacellaria & 69 & 23.8 & 17 & 8.2 & 30 & 24.8 \\
Chrysomya rufifacies & 13 & 4.5 & 1 & 0.5 & 9 & 7.4 \\
Lucilias ericata & 12 & 4.1 & 2 & 1 & 0 & 0 \\
Lucilia eximia & 9 & 3.1 & 3 & 1.4 & 3 & 2.5 \\
Totales & 290 & 100 & 208 & 100 & 121 & 100 \\
\hline
\end{tabular}

En cuanto a la abundancia de especies por órgano, se registró en el cerebro la mayor cantidad de moscas Chrysomya megacephala con 187 en cerebro, 185 en hígado y 79 en corazón; seguido de Cochliomyia macellaria con 69 en el cerebro, 30 en corazón y 17 en hígado; Chrysomya rufifacies con 13 individuos en cerebro, 9 en el corazón y un individuo en hígado (Figura 2).

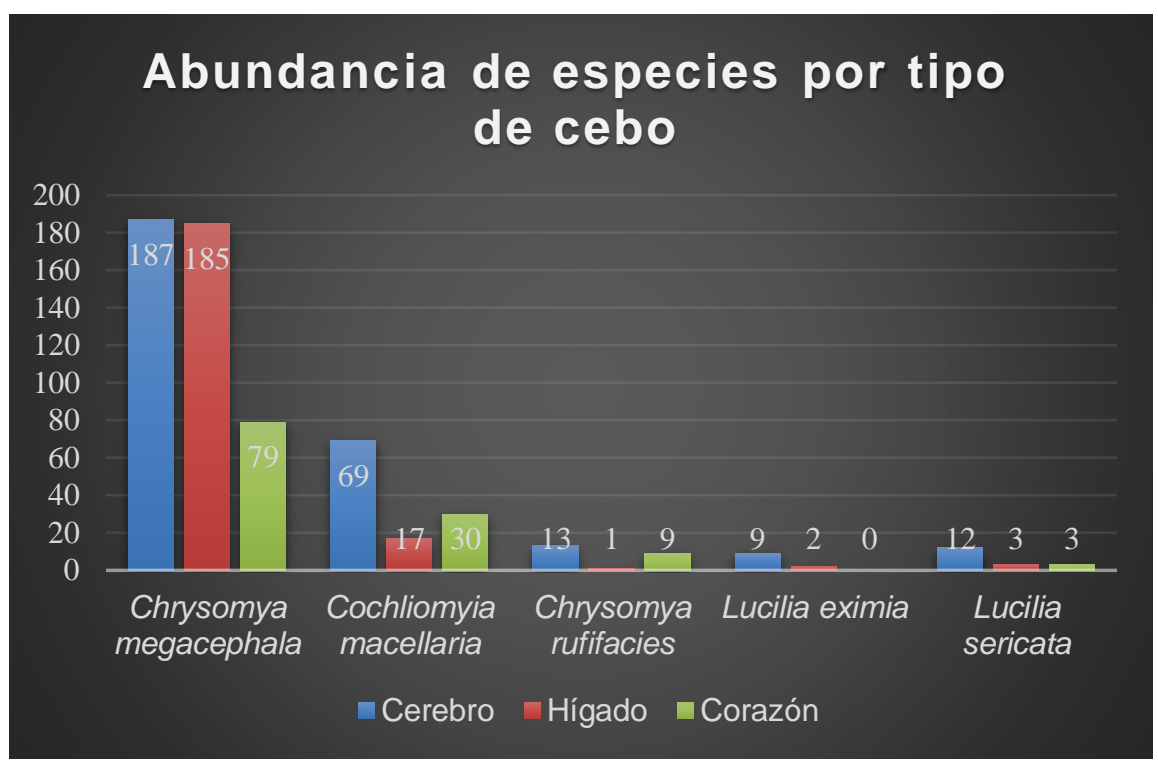

Fig. 2 Abundancia de especies por tipo de cebo 
Las especies Chrysomya megacephala y Cochliomyia macellaria fueron las más abundantes, ambas están correlacionadas por su índice de ponderación, por su peso del mismo signo. Mientras que las especies Chrysomya rufifacies, Lucilias ericata y Lucilia eximia tienen un menor peso en al análisis de correspondencia.

\section{DISCUSIÓN}

Los resultados coinciden de Rodríguez y Salazar, quienes realizaron ensayos con vísceras de res, reportaron a Chrysomya megacephala, Lucilia cuprina, Chrysomya albiceps y Cochliomyia macellaria como especies asociadas más abundantes. Otro estudio realizado por (Camacho, 2005) con cadáveres de cerdos, en Colombia, reportó a Calliphora vecina, Cochliomyia macellaria y Lucila sericata como las especies más abundantes.

Con frecuencia los géneros de estas especies están entre los que participan de la oviposición y consumo de los cuerpos en descomposición, la diferencia entre las especies se puede deber al tipo de ecosistema, la localización geográfica, área geográfica y a la extensión del estudio (Anderson \&VanLaerhoven. 1996; Camacho, 2003; Amat et al., 2008; De Sousa y VonZuben, 2012; Montoya, 2009). Sin embargo, este dominio en la explotación de las vísceras está determinado por la abundancia relativa de las especies y la proporción en que se encuentran sus poblaciones en el medio. De acuerdo con Paraluppi, (1996) el género Chrysomya, es dominante sobre el género Cochliomyia, lo que puede deberse a diversos factores como su rápida adaptabilidad a ambientes de claros, gran asociación con los ambientes urbanos y altos índices sinantrópicos. Las asociaciones de estos factores podrían determinar el éxito reproductivo de una o varias especies y convertirlas en eficientes colonizadoras y explotadoras de las carcasas.

Se ha demostrado que las moscas del género Chrysomya suelen ser los primeros en llegar a la carroña, a menudo en cuestión de horas después de la muerte, pues prefieren carroñas en las primeras etapas de la descomposición (Byrd y Castner, 2010). 
En términos generales, las moscas mostraron mayor preferencia por la víscera, el cerebro, seguido del hígado y el corazón. Esto pudiera deberse en parte a la consistencia de estos ya la microflora bacteriana dominante en su estado de descomposición. La especie Chrysomya megacephala fue la más abundante en el área de estudio, coincidiendo esta abundancia con su mayor frecuencia en las vísceras. En el cerebro se capturaron 187 especímenes, seguido muy de cerca del hígado con 185 especímenes y menor cantidad el corazón, con 79 especímenes (Cuadro 1). Nuestro resultado coincide en el área rural con los de Buitrago et al.,(2012) quienes estudiaron en las áreas intervenidas (urbana y rural), emplearon vísceras de pescado, reportaron que las mayores capturas fueron de Chrysomya megacephala con 3892 especímenes y Cochliomyia macellaria con 1098 ejemplares.

Otros autores también reportaron resultados parecidos Carrillo et al., (2015) reportaron a las especies Chrysomya albiceps con 407 ejemplares Cochliomyia macellaria con 240 ejemplares y Chrysomya megacephala con 95 ejemplares. Sus resultados se parecen a los nuestros, la diferencia se debe a la especie Chrysomya albiceps que no ha sido reportado en nuestro país. No obstante, existe cierta similitud en cuanto a las especies, pero con una ligera diferencia en cuanto orden en que aparecen y a sus cantidades. Rodríguez y Salazar (2014) emplearon vísceras de res, encontrando que la especie Chrysomya megacephala con 63 especímenes obtuvo la mayor captura, seguido de Lucilia cuprina con 13 especímenes, Chrysomya albiceps con nueve ejemplares y Cochliomyia macellaria con un ejemplar.

También De Sousa y VonZuben (2012) emplearon vísceras de hígado de res y sardina como atrayentes reportaron a las especies Lucilia eximia con 794 especímenes, Chrysomya albiceps Wiedmann, con 145 especímenes y Chrysomya megacephala con 134 especímenes. Estas diferencias en cuanto a la cantidad de especímenes y al orden en que dominan las especies, se pueden deber al tipo de cebo empleado para la atracción de las moscas, a los ecosistemas y a la metodología usada. Aunque, Tomberlin y Adler (1998) y Oliveira-Costa et al., (2001), señalan que las variaciones estacionales influyen significativamente en 
la abundancia de algunas especies.

Barbosa et al., (2010) estudiaron en carcasas de cerdos y reportaron que $C$. megacephala fue el Calliphoridae más abundante en todo el experimento, con el $96.3 \%$ de los individuos recolectados en el verano.

Nuestros resultados coinciden con lo obtenido por Camacho (2005) quién encontró que Cochliomyia macellaria fue también la segunda más abundante en su área de estudio. Esta especie según (Nuorteva, 1963) se encuentra mayormente en ambientes urbanos, debido a su preferencia por los asentamientos humanos. Gomes, (2009) también registró esta especie en los estados de hinchazón y descomposición activa. El orden y la cantidad en que se registró esta especie en las vísceras con firma lo que se ha observado en otras ocasiones, donde aparece como especie una exclusiva o como colonizadora primaria en los cuerpos en descomposición, en cadáveres frescos (Garcés et al., 2004).

La tercera especie más abundante fue Chrysomya rufifacies más frecuentemente capturada en las vísceras (Figura 3). Nuestros resultados coinciden en parte con los de (Rodríguez y Salazar, 2014, quienes encontraron con esta especie mostró baja preferencia por los cebos (pulmón e hígado). En tanto que Kyerematen et al., (2013) reportaron el arribo de esta especie durante el estado fresco de la descomposición. Vanegas, (2007) señala que esta especie es considerada una invasora secundaria en las carcasas. Lee et al., (2004) reportaron esta especie en 132 cadáveres, lo que indica que es una potencial indicadora forense. En nuestro país las larvas de esta especie han sido recuperadas de cadáveres humanos y compitiendo con las de Chrysomya megacephala.

Por otro lado, la especie Lucilia sericata mostró menor preferencia por las vísceras o que sus poblaciones naturales fueran muy bajas en el área de estudio. De acuerdo con (Ash y Greenburg, 1975) es una mosca es cosmopolita, con un alto grado de preferencia por vivir cerca de las viviendas. Según algunos autores (Figueroa y Linhares, 2002; Camacho, 2003; Saigusa et al., 2009; Pinilla et al., 2010) es 
referenciada como una de las primeras colonizadoras de cadáveres.

Por último, la especie Lucilia eximia fue la que registró la captura más baja. Contrario a lo encontrado por De Sousa y VonZuben (2012) quienes obtuvieron grandes cantidades. De acuerdo con Jirón, (1979) es una especie común en la región Neotropical y una de las más frecuentes en Costa Rica. Se ubica primordialmente en áreas urbanas, donde se alimenta de carroña, frutas y desechos orgánicos diversos ((Montoya et al., 2009; Wolff et al., 2010). Según (Giraldo et al., 2011; Andrade et al., 2005) es una especie de importancia forense debido a su asociación con cuerpos en descomposición.

Las especies Chrysomya megacephala y Cochliomyia macellaria fueron las más abundantes en nuestra área de estudio, de acuerdo con el análisis de correspondencia. Ambas están positivamente correlacionadas ya que su índice de ponderación es positivo (Figura 4), en una proporción de (3.8:1) (Cuadro 1). Lo cual confirma la atracción que mostraron los diversos órganos. A diferencia de las especies Chrysomya rufifacies, Lucilia sericata y Lucilia eximia que mostraron menor signo negativo, por sus bajas poblaciones número (Figura 4), con una proporción de (1.2:1.6:1).

En nuestro estudio se encontró que la especie Chrysomya megacephala mantiene poblaciones abundantes en el área, lo que le da ventaja competitiva sobre las especies Chrysomya rufifacies, Cochliomyia macellaria y Lucilia sericata. Contrario a lo que ocurre en otros sitios como Boquete con condiciones ambientales diferentes donde se ha observado un claro dominio de Cochliomyia macellaria, sobre Chrysomya megacephala y Chrysomya rufifacies (Garcés y Morales, 2017).

Lo importante de nuestro estudio es que bajo ciertas condiciones ambientales cualquiera de las especies reportadas, juegan un papel importante en la ciencia forense, en el establecimiento del IPM, en las investigaciones criminales. 


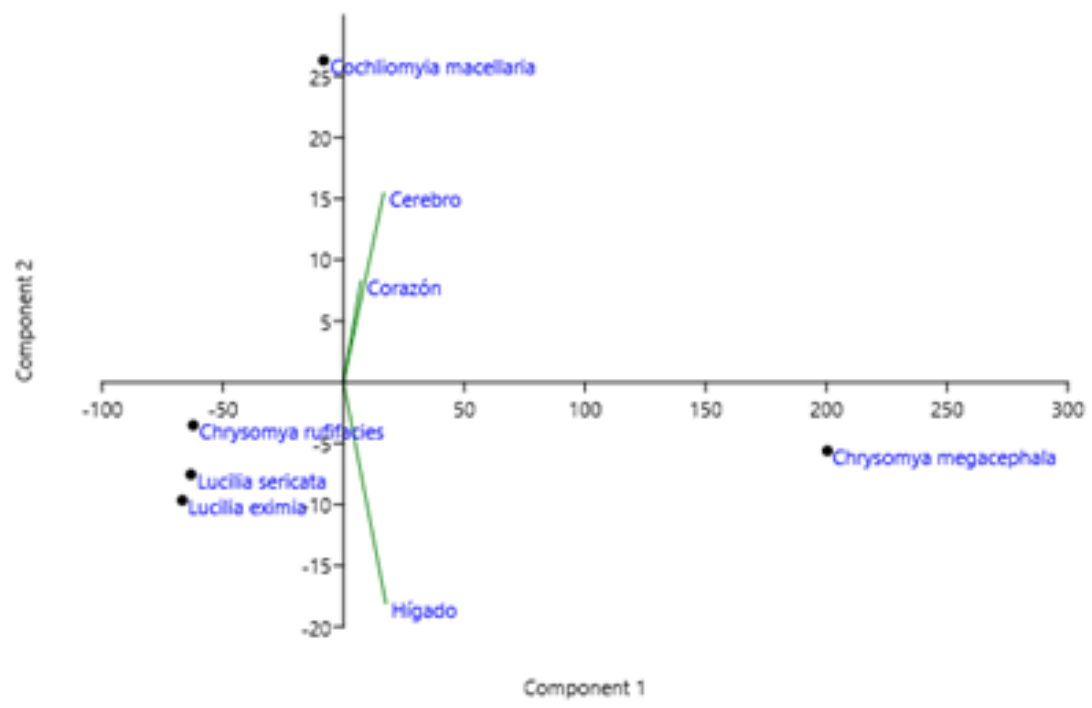

Fig. 4 Análisis de correspondencias (CPA) que muestra la tendencia de las cinco especies de la familia Calliphoridae

\section{CONCLUSIONES}

En nuestra área de estudio, las especies más abundantes en la misma fueron Chrysomya megacephala, Cochliomyia macellaria, por lo que, ambas pudieran ser consideradas buenas indicadoras forenses del IPM, por su notable abundancia en las vísceras.

Las moscas mostraron mayor preferencia por el cerebro, seguido por el hígado y por último por el corazón, lo que pudiera deberse al grado de consistencia de los tejidos y a los diferentes grados de descomposición.

Nuestra área de estudio mostro bastante similitud respecto a las especies encontradas y reportadas en las áreas urbanas del país. 


\section{AGRADECIMIENTOS}

A la Secretaria Nacional de Ciencia, Tecnología e Innovación (SENACYT), por el apoyo económico ofrecido durante esta investigación.

\section{REFERENCIAS}

Amat, E., M. Vélez y M. Wolff. 2008. Clave ilustrada para la identificación de los géneros y las especies de califóridos (Diptera: Calliphoridae) de Colombia. Caldasia, 30: 231-244.

Amendt, J., C. S. Richards, C.P. Campobasso, R. Zehner y M. J. Hall. 2011. Forensic entomology: applications and limitations. Forensic Science Medicine and Pathology; doi: 10.1007/s12024-010-9209-2.

Anderson, G. S. y S. L. VanLaerhoven. 1996. Initial studies on insect succession on carrion in southwestern British Columbia. J. Forensic.Sci. 41: 617-625.

Andrade, H. T., A. A. Varela- Freire, M. J. Batista y J. F. Medeiros. 2005. Calliphoridae (Diptera) Coletados em Cadáveres Humanos no Rio Grande do Norte. Neotropical Entomology. 34:855-856.

Ash, N. y B. Greenberg, 1975. Developmental temperature responses of the sibiling species Phaenicia sericata and Phaenicia pallescens. Annals of the Entomolgical Society of America, 68:197-200.

Buitrago, Y., R. Miranda y S. Bermúdez. 2011. Calliphoridae (Insecta: Diptera) de Ciudad de Panamá, Panamá, con énfasis en la distribución actual del género Chrysomya Robineau-Desvoidy 1830. Boletín de la Sociedad Entomológica Aragonesa, 49: 303-307.

Byrd, H., J. y J. L. Castner. 2010. Insects of forensic importance. En: Byrd y Castner (Eds.). Forensic Entomology. The Utility of Arthropods in Legal Investigations. Second edition. CRC Press, Boca Raton, FL, USA. 681 pp. 
Camacho, G. 2005. Sucesión de la entomofauna cadavérica y ciclo vital de Calliphora vicina (Diptera: Calliphoridae) como primera especie colonizadora, utilizando cerdo blanco (Sus scrofa) en Bogotá. Revista Colombiana de Entomología. 31:189-197.

Camacho, G. 2003. Sucesión de la entomofauna cadavérica y ciclo vital de Calliphoravicina (Diptera: Calliphoridae), como primera especie colonizadora, utilizando cerdo blanco (Sus scrofa) en Bogotá. Rev. Colomb. Entomol, 31:189-127.

Carrillo, G. E, M. A. Alcántara, C. C. Arias, C. Infante y M. Villacorta. 2015. Entomofauna de interés forense asociada a restos cadavéricos de cerdos (Sus scrofa L.), expuestos a condiciones de campo en Lambayeque - Perú. Revista de Perú. Entomología. 50: 1-11

De Sousa, C. R y C. J. VonZuben. 2012 Diversity and Synanthropy of Calliphoridae (Diptera) in the Región of Rio Claro, SP, Brazil Neotropical Entomologia 41:243-248.

Figueroa, L \& A. Linhares. 2002. Sinantropía de los Calliphoridae (Diptera) de Valdivia. Chile. Neotrop. Entomol, 31:233-239.

Garcés P. A. y W. P. Morales. 2017. Informe de una muerte traumática ocurrida en "potrero seco", provincia de Chiriquí, resuelto mediante el uso de la entomología forense. Tecnociencia, 19: 33-46

Giraldo, P. A., S. I. Uribe \& A. R López. 2011. Análisis de secuencias de ADN mitocondrial (Cytb y ND1) en Lucilia eximia (Diptera: Calliphoridae). Revista Colombiana de Entomología, 37: 273-278.

Gomes, G. 2009. A preliminary study of insect fauna on pig carcasses located in sugarcane in winter in southeastern Brazil. Medical and Veterinary Entomology, 23: 155-159.

Guimarães, J. H., N. Papavero \& A.P. Prado. 1983. As Mí́asesna Região Neotropical. Revista Brasileira de Zoologia, 1:239-416. 
Jirón, L. F., 1979. Sobre moscas califóridas de Costa Rica (Díptera: Cyclorrhapha). Brenesia 16: 221-223.

Kyerematen, R.A., B.A. Boateng, M. Haruna \& V. Eziah. 2013. Decomposition and insect succession pattern ofex posed domestic pig (Sus scrofa L.) carrion. Journal of Agricultural and Biological Science, 8: 756-765.

Lee, H. L., M. Krishnasamy, A. G. Abdullah, J. Jeffery 2004. Review of forensically important entomological specimens in the period 19722002. Tropical Biomedicine (supplement 1): 69-75.

Mariluis, J. C. \& J. A. Schnack. 2002. Calliphoridae de la Argentina. Sistemática, Ecología e Importancia Sanitaria (Diptera, Insecta). En: Actualizaciones en artropodología sanitaria Argentina Ed. Salomón, O.D. Serie Enfermedades Transmisibles, Publicación Monográfica Fundación Mundo Sano, 2: 23-37.

Montoya, A., J. D. Sánchez \& M. Wolff. 2009. Sinantropía de Calliphoridae (Diptera) del Municipio La Pintada, Antioquia Colombia. Revista Colombiana de Entomología, 35: 73-82.

Nuorteva, P. 1963. Synanthropy of blowflies (Dipt.Calliphoridae) in Finland. Annales Entomologici Fennici, 29: 1-49.

Oliveira-Costa, J., C. A. Mello y S. M. López. 2001. Dípteros muscóides associados a cadáveres humanos no local da morte no estado do Rio de Janeiro. Brasil. Bol. Mus. Nac., N.S., Zool. 464: 1-6

Paraluppi, N. D. 1996. Calliphoridae (Diptera) da Bacia do Alto Rio Rucu, Amazônia Central, Brasil. Revista Brasileira de Zoologia, 13: 553-559.

Pinilla B., Y. Acuña, B. Cortes, R. Díaz, A. Segura \& F. J. Bello. 2010. Características del ciclo biológico de Lucilia sericata (Meigen, 1826) (Diptera: Calliphoridae) Sobre dietas diferentes. Rev. U.D.C.A Act. \& Div. Cient, 13: 153-161. 
Rodríguez, J. N. y J. L. Salazar. 2014. Sucesión de la entomofauna cadavérica a partir de un biomodelo con vísceras de res. Revista de la Facultad de Ciencias de la Salud. Universidad de Carabobo. 18: 35-39.

Rognes, K. 1997. The Calliphoridae (Blowflies) (Diptera: Oestroidea) are not a monophyletic group Cladistics 13: 27-66.

Saigusa, K., M. Matsumasa, Y. Yashima \& Y. Yoichi. 2009. Practical applications of molecular biological species identification of forensically important flies. Legal Medicine, 11:344-347.

Tomberlin, J. K. y P. H. Adler 1998. Season alcolonization and decomposition of rat carrion in water and on land in open field in South Carolina. J MedEntomol 35: 704-709.

Vanegas, S. Y. 2007. Efectos de la temperatura sobre el desarrollo de Chrysomya rufifaciesy Cochliomyia macellaria (Diptera: Calliphoridae), dos especies importantes para la entomología forense en Puerto Rico. Tesis Universidad de Puerto Rico. 98pp.

Whitworth, T. 2006. Claves para géneros y especies de moscas califóridas (Diptera: Calliphoridae) de América al norte de México. Proceedings of the Entomological Society of Washington, 108: 689725.

Wolff, M., A. Uribe, A. Ortiz \& P. Duque. 2001. A preliminary study of forensic entomology in Medellín, Colombia. Forensic Science International; 120: 53-59.

Zump, F. 1965. Myiasis in man and animals in Old World. Butterworths, London: 267 p.

Recibido 11 de febrero de 2020, aceptado 11 de marzo de 2020. 